\title{
Update on the CDC National Syndromic Surveillance Program
}

\author{
Paula Yoon and Michael Coletta* \\ Centers for Disease Control and Prevention, Atlanta, GA, USA
}

\section{Objective}

Inform conference attendees about the CDC National Syndromic Surveillance Program (NSSP), various program-related projects and who is working on them, what was accomplished during the past year, and NSSP-development plans for the future.

\section{Introduction}

The Public Health Security and Bioterrorism Preparedness and Response Act of 2002 mandated establishing an integrated national public health surveillance system for early detection and rapid assessment of potential bioterrorism-related illness. In 2003, CDC created and launched the BioSense software program. At that time, CDC's focus was on rapidly developing and implementing Web-based software to collect hospital emergency department data for analysis to detect and monitor syndromes of public health importance. During the ensuing decade, BioSense evolved and now is part of CDC's renamed National Syndromic Surveillance Program (NSSP). The broader vision of NSSP aims to achieve two key goals: significantly improve technical capabilities for collecting and analyzing syndromic surveillance data, and to create and facilitate opportunities for collaboration among local, state, and national public health programs. Through NSSP, the syndromic surveillance community can be strengthened by access to improved technical capacity and to best-practices knowledge sharing among syndromic surveillance professionals. These NSSP initiatives can help the nation-wide public health community strengthen situational awareness and enhance response capability to hazardous events. NSSP encompasses people, partners, policies, information systems, standards, and resources. Session attendees will learn more about NSSP, its growing group of partners, what the program is doing now, and its future.

\section{Description}

The 2014 CDC Surveillance Strategy calls for modernizing its health surveillance systems. Through a host of improvements, CDC; its state, tribal, local, and territory public health partners; and the public health community-at-large will have better data and information to help inform decisions. The panel will discuss the BioSense Platform, formerly known simply as BioSense, which is one of the four national surveillance programs identified for transformation in the CDC Surveillance Strategy. The BioSense Platform is now a component of NSSP, which was launched last year by CDC's Division of Health Informatics and Surveillance.

The panel will discuss how the NSSP staff is upgrading the BioSense Platform in three main areas:

1) Data Quality: Improving data management, quality, and representativeness

2) Technology: Upgrading BioSense Platform technological capacity with better tools for data collection, processing, and analyses

3) Partner Engagement: Strengthening the syndromic surveillance Community of Practice to promote data sharing that will further the science and practice of syndromic surveillance

The panel will consist of CDC staff members who are leading these efforts and representatives from the BioSense Governance Group (BGG), an organization representing public health jurisdictions participating in NSSP. The BGG collaborates with CDC to develop ways to improve BioSense Platform performance including the tools and services it hosts. The panel will provide updates on current and near-term NSSP activities, describe recent enhancements to the program, and engage the audience in discussions about ways to improve the utility of syndromic surveillance at all levels of the public health enterprise. Discussion topics might include the anticipated deployment of new tools on the BioSense Platform such as ESSENCE, SAS, and R Studio Professional; identifying technical assistance, training, and support needs of local and state programs; data work flow and ways to improve data quality; strengthening the NSSP Community of Practice initiative by increasing local-and statelevel engagement; and ideas and topics for collaborations among jurisdictions and with CDC.

\section{Audience Engagement}

Director of CDC's Division of Health Informatics and Surveillance Paula Yoon, ScD, will introduce the NSSP team (Program Manager, BioSense Platform Lead, Data Quality Lead, Partner Engagement Lead, and BioSense Governance Group (BGG) partners) and provide historical perspective on BioSense from inception in 2003 to its current role in NSSP. Then NSSP team members will describe work underway and goal accomplishments in the three modernization focus areas identified earlier. The NSSP program manager will offer a vision for the future. The BGG partners will describe their roles in representing the public health syndromic surveillance community. They also will facilitate an audience discussion intended to discover others' viewpoints and recommendations.

\section{Keywords}

NSSP; BioSense Platform; CDC

\section{*Michael Coletta}

E-mail: mac0@cdc.gov 